\section{Canada}

Research

Branch
Direction générale de la recherche

Contribution 1983-15F

\title{
Lutte rationnelle contre les ravageurs des pommiers au Québec
}
630.72
C759
C $83-015$
$\mathrm{fr}$.
c. 3

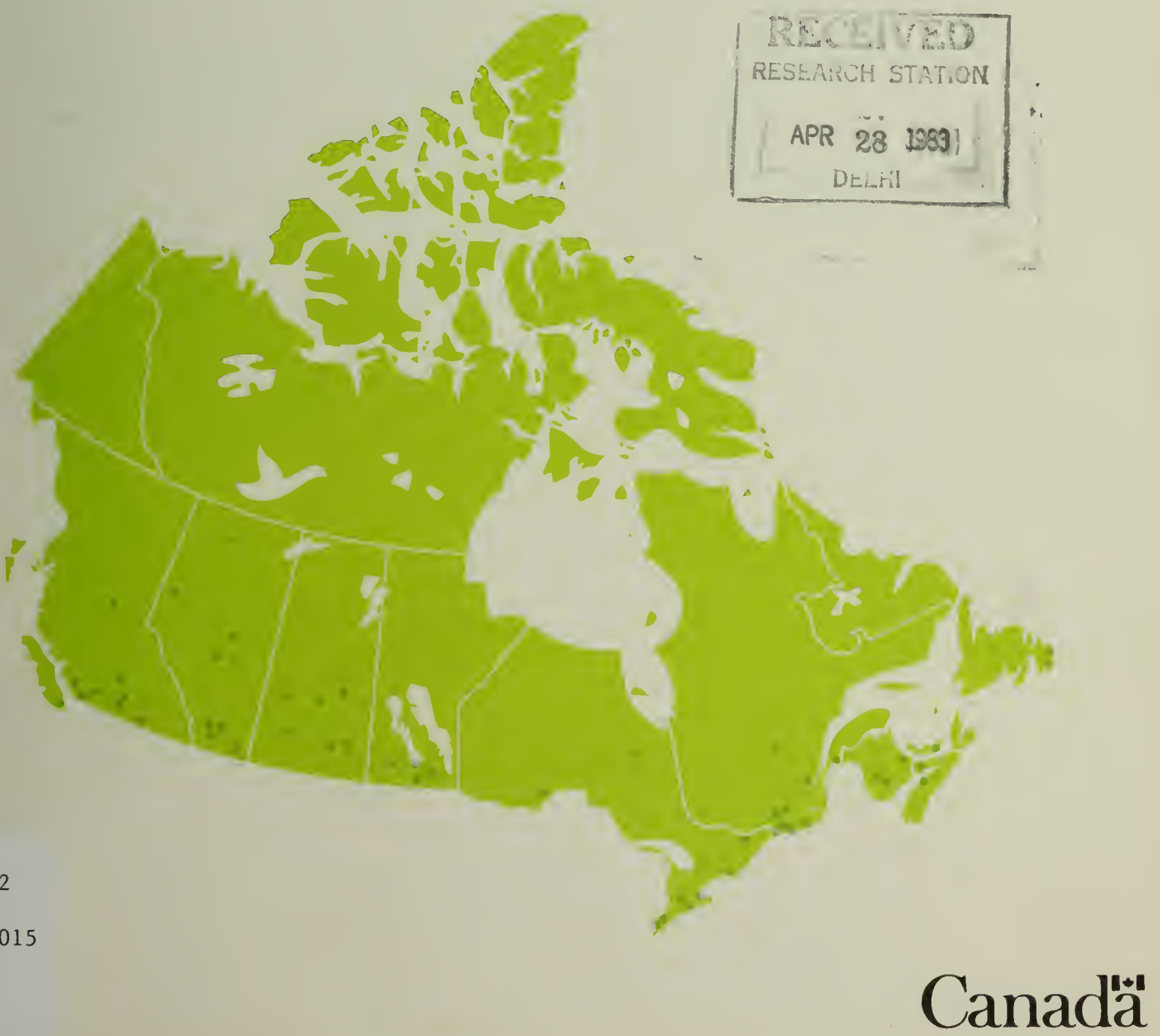


Sur la couverture, les points sur la carte indiquent les établissements de recherche d'Agriculture Canada 


\section{Lutte rationnelle contre les ravageurs des pommiers au Québec}

RODOLPHE OMER PARADIS

Station de recherche

Saint-Jean-sur-Richelieu (Québec)

Bulletin technique $n^{\circ} 16$ revisé

Direction générale de la recherche Agriculture Canada 1983 
On peut obtenir des exemplaires de ce bulletin à la Station de recherche

Direction générale de la recherche

Agriculture Canada

C.P. 457

Saint-Jean-sur-Richelieu (Québec)

J3B 6 Z8

Production du Service aux programmes de recherche

CMinistre des Approvisionnements et Services Canada 1983

Also available in English under the title

Pest management approach in Quebec apple orchards 
The appearance and behaviour of phytophagous insects and mites occurring in apple orchards in southwestern Québec are presented along with the phenological development of the cultivar McIntosh coupled with degree-day summations. Natural enemies of these pests are also mentioned.

Chemical control measures require five pesticide treatments applied at different apple bud development stages and pre-determined dates. These treatments are directed against the major pests, $v i z$. the European red mite, Panonychus uImi (Koch), the apple maggot, Rhagoletis pomone Zla (Walsh), the bugs Lygus Zineolaris ( $\mathrm{P}$. de B.), Lygocoris communis (Knight) and Lygidea mendax Reut., the green fruitworm, Or thosia hibisci (Guenée), and the plum curculio, Conotrachelus nenuphar (Hbst.). Monitoring techniques are being evaluated with the aim of reducing pesticide treatments in apple orchards.

Note Contribution No. J. 847, Research Station, Research Branch, Agriculture Canada, Saint-Jean-sur-Richelieu, Québec.

Paper presented at a colloquium on horticulture held at the International Floralies, Montreal, Quebec, August 20, 1980 

Résumé

Les insectes et les acariens ravageurs présents dans les pommeraies du sud-ouest du Québec sont passés en revue en fonction de leur comportement relié au développement phénologique du cultivar McIntosh ainsi qu'à des constantes thermiques. Il est fait mention également des principaux ennemis naturels de ces ravageurs.

La lutte chimique préconisée comporte cinq traitements appliqués à des stades-repères et à des dates prédéterminés; ces traitements sont dirigés contre les ravageurs prépondérants qui sont le Tétranyque rouge du pommier, Panonychus ulmi (Koch), la Mouche de la pomme, Rhagoletis pomonelza (Walsh), les Punaises Lygus Zineolaris (P. de B.), Lygocoris communis (Knight) et Lygidea mendax Reut., la Noctuelle du fruit vert, Orthosia hibisci (Guenée), et le Charançon de la prune, Conotrachelus nenuphar (Hbst.). Des essais de piégeage sont en cours et visent essentiellement à réduire le nombre de traitements insecticides.

Note Contribution no J. 847 de la Station de Recherches, Direction générale de 1a Recherche, Agriculture Canada, Saint-Jean-surRichelieu, Québec.

Communication présentée 1e 20 août 1980 , au Colloque International sur l'Horticulture patroné par les Floralies de Montréal, Québec 
Dès le début, je tiens à souligner que cette communication a été préparée avec l'aide de collègues qui oeuvrent dans le domaine de la protection des vergers du Québec, nommément monsieur Marcel Mailloux du ministère de l'Agriculture, des Pêcheries et de l'Alimentation du Québec, ainsi que messieurs Irénée Rivard et Noubar Bostanian du ministère de 1 'Agriculture du Canada. J'ai aussi bénéficié de certaines informations fournies par monsieur Guy Boivin, étudiant à l'Université McGill, qui poursuit des études sur le complexe des Punaises phytophages des pommeraies et par monsieur Denis Bouchard, de 1 'Université du Québec à Montréal, dont les recherches portent sur les pucerons du pommier.

La première partie de cette communication traite de l'entomofaune des vergers du sud-ouest du Québec telle qu'elle se présente au cours d'une saison, d'abord en relation avec les stades phénologiques des pommiers du cultivar McIntosh, nettement prédominant dans nos vergers, puis en relation avec les mois du calendrier pour le reste de la saison. A chacun de ces stades, il est fait mention des degrés-jours calculés à partir du seuil de $5^{\circ} \mathrm{C}$ et selon la simple méthode (maximum + minimum/2) $-50 \mathrm{C}$. Ces constantes thermiques ont été cumulées au cours des dix dernières années.

L'entomofaune comprend d'une part les ravageurs et, d'autre part, quelques-uns des principaux ennemis naturels. Etant donné le très grand nombre de ravageurs qui infestent les pommiers, nous les avons divisés en trois catégories: les ravageurs prépondérants, responsables de pertes économiques dans la plupart des vergers à défaut de traitements; les ravageurs secondaires qui occasionnent localement et épisodiquement de sérieux dégâts et les ravageurs occasionnels qui, le plus souvent, causent des dégâts négligeables.

Dans la deuxième partie de la communication, il sera question du programme de lutte et de dépistage mis de 1 'avant au cours des dernières années et que $l^{\prime}$ on tente de préciser et d'améliorer toujours davantage. 
I - ENTOMOfAUNE DES VERGERS DE POMMIERS EN FONCTION DE LA PHÉNOLOGIE STADE DORMANT AU PRINTEMPS

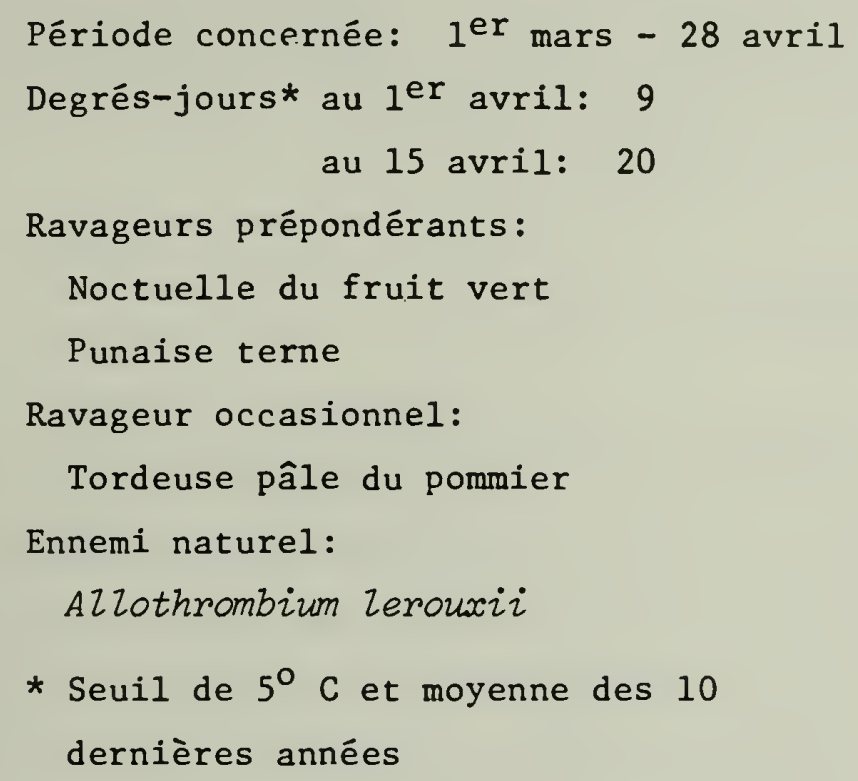

Lorsque les pommiers sont encore au stade dormant au printemps (P1. 1, fig. 1), certains insectes sortent déjà de leur hibernation et reprennent leur activité. C'est le cas précisément de la Noctuelle du fruit vert, Orthosia hibisci (Guenée), de la Punaise terne, Lygus Zineolaris (P. de B.), deux insectes classés présentement comme ravageurs prépondérants dans les pommeraies du Québec, c'est-à-dire pouvant occasionner des pertes économiques dans un très grand nombre de vergers si l'on néglige de faire les traitements appropriés.

Noctuelle du fruit vert

En 1980, la première capture de papillons de la Noctuelle du fruit vert, effectuée à l'aide de pièges à phéromones, est en date du 10 avril, soit une quinzaine de jours avant la période du débourrement. Les papillons de cette espèce sont de couleur gris beige, avec tête et thorax densément recouverts de poils et des ailes d'une envergure d'environ $40 \mathrm{~mm}$ (Pl. II, fig. 12). Ils voltigent dans les vergers ordinairement jusqu'à la mi-mai. 
Les premiers adultes de la Punaise terne ont été capturés le 15 avril en 1980. Ce sont de petits insectes brun terne, de forme ovale, qui mesurent $6 \mathrm{~mm}$ de longueur et qui s'envolent rapidement lorsqu'ils sont inquiétés (P1. II, fig. 3).

Tordeuse pâle du pommier

La Tordeuse pâle du pommier, Pseudexentera mali Free., est la seule tordeuse des vergers qui, selon nos observations, s'attaque uniquement aux pommiers, les autres tordeuses infestant à l'occasion différentes essences forestières. Elle doit son nom à la couleur blanchâtre des larves que $l^{\prime}$ on trouve bien dissimulées dans les pousses terminales des rameaux. Nous avons déjà observé les adultes de $P$. mali voltigeant dans les vergers les 8 et 9 avril alors que la température maximale journalière était de $12^{\circ} \mathrm{C}$ et que la neige au sol n'était pas entièrement disparue.

L'Acarien AZZothrombium Zerouxii Moss (P1. IV, fig. 1)

Parmi les rares entomophages dans les vergers à cette période de 1 'année, il faut mentionner l'acarien $A$. Zerouxii de 3,3 $\mathrm{mm}$ de longueur et d'un rouge velouté qui est souvent abondant immédiatement après la fonte des neiges. On le trouve dissimulé dans les feuilles mortes sur le sol et, très vorace, il s'attaque aux oeufs et autres formes de plusieurs espèces d'acariens et d'insectes.

\section{DÉBOURREMENT}

Le débourrement des pommiers, appelé en anglais 'green tip" et que l'on traduit souvent par "bouton vert" ainsi que par "pointe verte" constitue un stade de première importance (P1. I, fig. 2). En plus d'être le signe apparent du départ de la végétation, c'est à partir de cette époque que la tavelure, Venturia inaequalis (Cke.) Wint., peut s'implanter sur les 
pommiers et que bon nombre de ravageurs manifestent leur présence. Dans le sud-ouest du Québec, le débourrement le plus hâtif des pommiers McIntosh a été noté le 16 avril et le plus tardif, le 13 mai; la date moyenne est cependant le 29 avril.

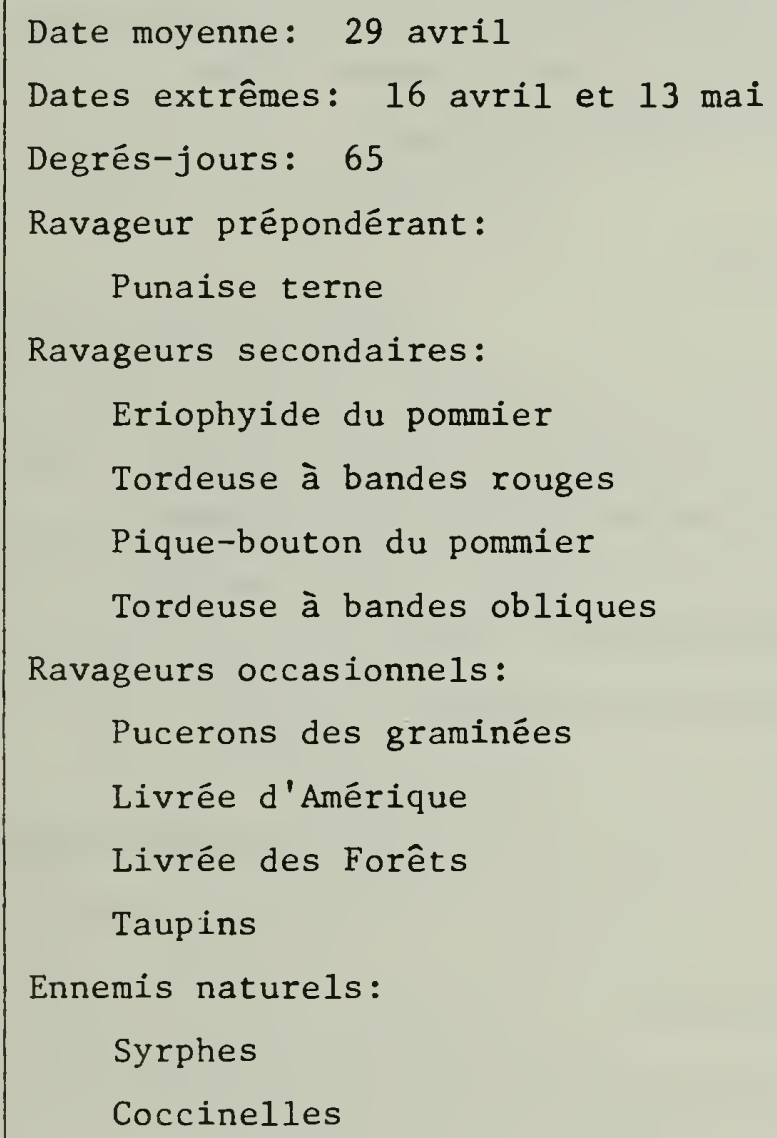

On retrouve la punaise terne qui, pour s'alimenter, pique les bourgeons floraux et les bourgeons végétatifs ce qui provoque d'abord des exsudations chez ces bourgeons puis leur dessèchement. Nous avons noté que, dans certaines plantations, la proportion de bourgeons ainsi détruits peut facilement être de 1 'ordre de $30 \%$. 
Eriophyide du pommier (P1. II, fig. 2)

L'Eriophyide du pommier, Aculus schlechtendali (Nal.), infeste de plus en plus fréquemment et de plus en plus sérieusement les vergers du Québec. Mesurant environ $0,07 \mathrm{~mm}$ de longueur, il est invisible à l'oeil nu, mais le roussissement du feuillage en cours de saison révèle facilement sa présence. Les femelles hibernantes appelées deutogynes quittent donc les écailles des bourgeons où elles ont passé l'hiver pour infester les tissus foliaires dès la période de débourrement.

Tordeuse à bandes rouges

Passant l'hiver à l'état de chrysalides dans des débris végétaux sur le sol, les premiers papillons de la Tordeuse à bandes rouges, Argyrotaenia velutinana (W1k.) sont capturés à l'aide de phéromone synthétique à 1 'époque du débourrement. Il s'agit de petits papillons d'environ 13 mr d'envergure dont les ailes antérieures sont traversées obliquement par une bande nuancée de rouge et de brun.

Les captures des adultes de la génération hibernante se prolongent jusqu'à la mi-juin. Il arrive que ces captures soient très abondantes, mais il faut souligner que l'espèce est polyphage et que les chenilles peuvent être plus abondantes, par exemple, sur des plantes du genre Apocynum sp. croissant dans certains vergers que sur les pommiers euxmêmes.

Tordeuse à bandes obliques et Pique-bouton du pommier (PI. III, figs 4 et 8 )

La Tordeuse à bandes obliques, Archips rosaceana (Harr:), et le Piquebouton du pommier, Spizonota ocezzana (D. \& S.), passent I'hiver à 1 'état de jeunes chenilles sur les arbres. Dès la période de débourrement ces chenilles reprennent activité et se dissimulent à l'intérieur des bourgeons pour se nourrir. Les chenilles de $A$. rosaceana sont vert 
jaunâtre avec tête foncée tandis que celles de $S$. ocelzana sont brun chocolat.

\section{Puceron des graminées}

Parmi les quatre espèces de pucerons que 1 'on rencontre sur les pommiers, le Puceron des graminées, Rhopalosiphum fitchii (Sand.), est le premier à faire son apparition. Les populations sont rarement assez abondantès pour nécessiter des traitements. Au stade du calice, apparaissent les formes ailées qui migrent sur les graminées.

\section{Livrée d'Amérique et Livrée des forêts}

C'est à la fin d'avril, à la période de débourrement, qu'éclosent les chenilles de MaZacosoma americanum (F.) et de MaZacosoma disstria Hbn. Les chenilles de chacune de ces deux espèces vivent en colonie, mais seules celles de $M$. comericanum se tissent une toile aux fourches des arbres. Dans les vergers entretenus, elles sont facilement éliminées par les traitements réguliers d'insecticides. Leur développement larvaire prend fin à la mi-juin.

\section{Taupins}

On appelle ainsi les adultes des vers fil de fer. Certains de ces adultes, notamment du genre Ctenicera spp., grugent les bourgeons des pommiers et s'attaquent aussi aux fleurs, après quoi ils abandonnent les pommiers. Il s'agit de ravageurs occasionnels.

\section{Symphes}

Dès I'ouverture des bourgeons, on peut observer des oeufs de Syrphes déposés sur les bourgeons infestés par le Puceron des graminées (P1. IV, figs 2 et 3 ). Les larves de ces Diptères sont tout au cours de la saison d'importants prédateurs des Pucerons. D'après les études conduites par 
monsieur Denis Bouchard, il y en aurait au moins sept espèces dans nos vergers.

Coccinelzes (P1. IV, fig. 3)

Au début de la saison, les Coccinelles sont nombreuses et se déplacent ici et là en quête de nourriture. On en a identifié huit espèces s'attaquant aux Pucerons.

\section{DÉBOURREMENT AVANCÉ}

Le débourrement avancé se caractérise par des feuilles qui ont au moins $12 \mathrm{~mm}$ de longueur. Les pomologistes américains désignent d'ailleurs ce stade par l'expression "half-inch green". Enfin, on dénomme souvent ce stade "bouton vert avancé" ou encore "oreilles de souris" (P1. I, fig. 3). Les pommiers atteignent ce développement à la date moyenne du 2 mai, tandis que les dates extrêmes sont d'une part le 20 avril et, d'autre part, le 20 mai. Le nombre de degrés-jours alors accumulés est d'environ 85 unités. A ce stade phénologique des pommiers, apparaissent dans les vergers les premières fleurs de Pissenlit, Taraxacum officinale et de Trilles, Trizzium grandiflomm et $T$. erectum.

Au débourrement avancé, on retrouve les ravageurs déjà mentionnés auxquels viennent s'ajouter le Tétranyque à deux points, Tetranychus urticae Koch, le Puceron rose du pommier, Dysaphis plantaginea (Pass.), la Mineuse marbrée, Lithocolzetis bzancardelza (F.) et la Spongieuse, Lymantria dispar (L.).

\section{Tétranyque à deux points}

Le Tétranyque à deux points est un acarien cosmopolite et polyphage que $I^{\prime}$ on retrouve aussi bien en serre qu'en plein champ; il se classe comme ravageur secondaire des pommeraies au Québec. Les femelles hibernantes, de couleur jaune orange, font leur apparition habituellement au 
débourrement avancé. On les retrouve alors sur les plantes herbacées constituant le parterre des vergers, notamment sur le Lychnis blanc, Lychnis aZba. Ce n'est qu'à la fin de juin ou au début de juillet, à l'occasion d'une sécheresse ou à la suite du fauchage des plantes de couverture, que le Tétranyque à deux points envahit les pommiers. L'espèce complète cinq ou six générations dans le sud-ouest du Québec.

Date moyenne: 2 mai

Dates extrêmes: 20 avril et 20 mai

Degrés-jours: 85

Ravageurs secondaires:

Tétranyque à deux points

Puceron rose du pommier

Ravageurs occasionnels:

Mineuse marbrée

Spongieuse

Puceron rose du pormier (P1. III, figs 2 et 3 ).

Les pullulations du Puceron rose du pommier ont une incidence nettement sporadique. De plus, à la mi-juin, débute une forte migration des formes ailées vers des hôtes secondaires.

Mineuse marbrée

Le complexe des mineuses du pommier au Québec comprend les espèces suivantes: Lithocolletis malimalifoli,ella (Braun), L. scuderella (F. \& B.), L. crataegelza Clem. et L. blancardelza (F.). La Mineuse marbrée, L. blancardezza, est prédominante présentement; elle complète trois générations annuellement et passe l'hiver à l'état de chrysalide dans les feuilles mortes. Les adultes font leur apparition au débourrement avancé. Au printemps 1980, nous avons procédé pour la première fois au 
dépistage de L. blancardella au moyen d'une phéromone sexuelle synthétique.

\section{Spongieuse}

Ce déprédateur accuse une recrudescence sur les essences feuillues dans plusieurs régions du Québec. Dans certains vergers, au cours des dernières saisons, nous avons noté la présence des chenilles noirâtres et à longs poils de la Spongieuse qui se nourrissent très souvent à la surface supérieure des feuilles. Leur développement prend fin durant la deuxième quinzaine de juin. Toutefois, jusqu'à présent, ses proliférations en vergers sont occasionnelles.

\section{PRÉ-BOUTON ROSE}

A ce stade, on note l'apparition des boutons floraux, mais ceux-ci sont tous soudés les uns aux autres d'où l'expression anglaise de 'tight cluster' (P1. 1, fig. 4). La date moyenne de l'apparition du pré-bouton rose est le 9 mai, tandis que les dates extrêmes oscillent du 29 avril au 23 mai.

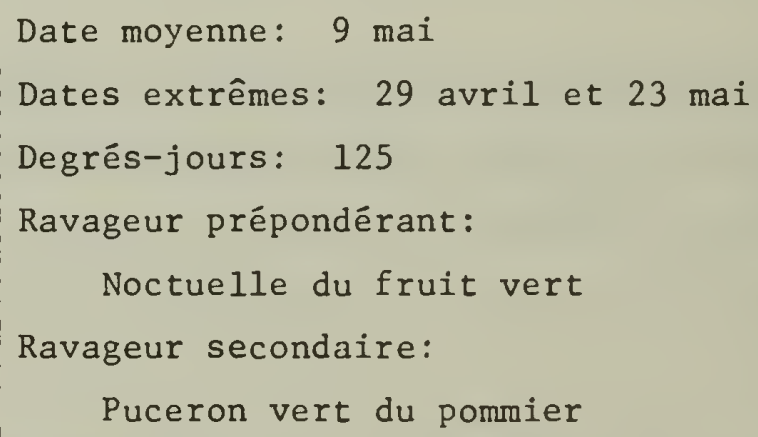

Date moyenne: 9 mai

Dates extrêmes: 29 avril et 23 mai

Degrés-jours: 125

Ravageur prépondérant:

Noctuelle du fruit vert

Ravageur secondaire:

Puceron vert du pommier

Noctuelle du fruit vert (P1. II, figs 11 et 13)

A cette époque de la saison débute l'éclosion des chenilles de la Noctuelle du fruit vert; leur développement se prolonge jusqu'à une 
quinzaine de jours après la chute des pétales. Ces chenilles s'attaquent aux bourgeons et aux pousses puis aux pommes nouvellement formées sur lesquelles elles pratiquent des morsures et des cavités profondes. Elles sont vert pâle, légèrement lignées de blanc; au terme de leur développement, elles mesurent de 30 à $40 \mathrm{~mm}$ de longueur.

Puceron vert du pommier (P1. III, fig. 1)

Au stade du pré-bouton rose, on note des colonies du Puceron vert du pommier, Aphis pomi De G, sur les pousses terminales. Cette espèce passe la saison entière sur le pommier. Les formes ailées qui apparaissent un peu plus tard contribuent à sa dispersion dans les vergers. Lorsque les populations deviennent très denses au cours de la saison, elles occasionnent de la fumagine sur le feuillage et sur les fruits.

\section{BOUTON ROSE}

A ce stade, les boutons floraux se détachent les uns des autres et, sur chacun des boutons, on perçoit une section des pétales nettement colorée de rose (P1. 1, fig. 5). Le stade du bouton rose prend place habituellement à la mi-mai; toutefois, la date la plus hâtive a été le 6 mai, et la plus tardive, le 29 mai. A ce stade, on compte 165 degrés-jours.

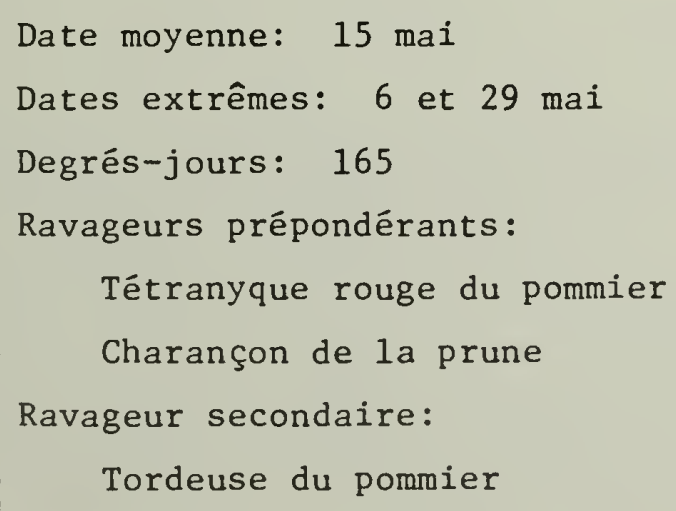


Tétranyque rouge du pomonier (P1. II, fig. 1)

Les oeufs du Tétranyque rouge du pommier, Panonychus ulmi (Koch), qui ont passé 1 'hiver sur 1 'écorce des branches et des troncs, éclosent en grand nombre chaque année au stade du bouton rose. Il convient de souligner que $P$. $u I m i$ est un des pires ravageurs des pommiers au Québec. Il pullule très facilement sur les arbres, pouvant ccmpléter six ou sept générations en une saison. Pour s'alimenter, il soutire les matières cellulaires des feuilles occasionnant leur décoloration; il réduit ainsi la vigueur de l'arbre et le développement des bourgeons à fruits; il peut enfin causer une chute prématurée des fruits. Le coût des traitements appliqués contre le Tétranyque rouge et autres acariens représentent environ $30 \%$ du coût total des traitements effectués contre l'ensemble des ravageurs.

Charançon de la prune (P1. II, fig. 8)

Les premiers adultes hibernants du Charançon de la prune, Conotrachelus nenuphar (Hbst.), font également leur apparition du stade du bouton rose. Malgré son nom, cet insecte est prépondérant dans les pommeraies du Québec et, n'ayant que très peu d'ennemis naturels, on doit recourir à des traitements insecticides pour protéger les pommes contre ses attaques.

Tordeuse du pomier (P1. III, fig. 9)

La Tordeuse du pommier, Archips argyrospilus (W1k.), se classe parmi les ravageurs secondaires. Les oeufs déposés en plaques sur l'écorce des arbres constituent le stade d'hibernation et éclosent principalement au stade du bouton rose. Les chenilles de couleur verte avec tête noire grugent les premiers éléments des tissus foliaires et les différents organes floraux; par la suite, à l'aide de soies, elles enroulent les feuilles en forme de fourreau où elles se dissimulent, dévorant ici et là le parenchyme foliaire, et s'attaquent aussi aux pommes nouvellement 
formées à proximité de leur abri. Ces dégâts causés aux fruits peuvent facilement être confondus avec ceux causés à la même époque par les chenilles de la Noctuelle et celles de la Tordeuse à bandes obliques (P1. II, fig. 13, P1. III, fig. 10).

\section{BOUTON ROSE AVANCÉ}

Ce stade est assez rapproché du précédent. Il s'en distingue par des pétales beaucoup plus développés qui demeurent cependant soudés les uns aux autres et qui affectent la forme d'un ballon teinté de rose et de blanc (P1. I, fig. 6). La date moyenne de l'apparition du bouton rose avancé est le 19 mai avec une accumulation de 200 degrés-jours. La date la plus hâtive a été observée le 16 mai et la plus tardive, le 31 mai.

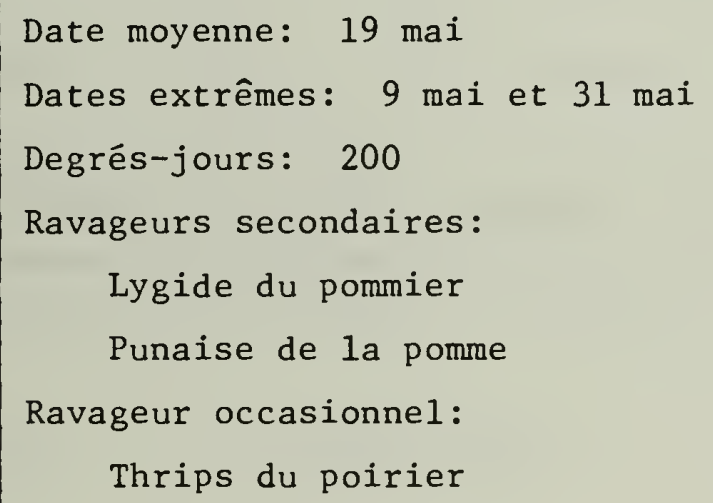

Le stade repère du bouton rose avancé demeure important dans les programmes de lutte contre la tavelure du pomier. Concernant les ravageurs, le développement des espèces déjà signalées se continue et à celles-ci viennent s'ajouter la Punaise de la pomme, Lygocoris communis (Knight), la Lygide du pommier, Lygidea mendax Reut. et le Thrips du poirier, Taeniothrips inconsequens (Uzel). 
Lygide du pormier et Punaise de la pomme

Ces deux espèces de Mirides passent l'hiver à l'état d'oeufs implantés dans les brindilles et dans les pousses terminales des rameaux. Les larves éclosent au stade du bouton rose avancé; les larves de la Lygide du pommier sont rouge vermilion et celles de la Punaise de la pomme, vert pâle. Les études en cours semblent démontrer que les attaques de ces ravageurs sur les pommes sont plus importantes qu'on le croyait.

Thrips du poirier

Malgré son appellation, cet insecte suceur infeste aussi les pommiers, pruniers, cerisiers, pêchers ainsi que certaines essences forestières. Les adultes sont de couleur noire et mesurent environ $1,5 \mathrm{~mm}$ de longueur. Ils s'introduisent dans les fleurs de pommier en voie de développement et, pour s'alimenter, piquent les différents organes provoquant ainsi le brunissement et la nécrose des tissus. De plus, les femelles causent d'autres lésions en implantant leurs oeufs dans les pétioles des fleurs et des fruits ainsi que dans les nervures médianes des feuilles. Les larves, de couleur blanc jaunâtre, soutirent les matières cellulaires à la façon des adultes, complètent leur développement en juin puis tombent au sol pour la nymphose. L'espèce est univoltine.

\section{PLEINE FLORAISON}

A ce stade, toutes les fleurs sont ouvertes (P1. I, fig. 7). La date moyenne de la pleine floraison est le $23 \mathrm{mai}$ avec accumulation de 240 degrés-jours. La date de floraison la plus hâtive a été notée le 13 mai et la plus tardive, le 5 juin.

C'est l'époque de la pollinisation; une étude récente sur le sujet évaluant 1.'importance des insectes en regard de certains autres agents pollinisateurs a été conduite dans une pommeraie du sud-ouest du Québec par mademoiselle Suzanne Pion et fera sous peu l'objet d'une publication. 
Date moyenne: 23 mai

Dates extrêmes: 13 mai et 5 juin

Degrés-jours: 240

Ravageurs prépondérants:

Tétranyque rouge du pommier

Charançon de la prune

Ravageurs occasionnels:

Hoplocampe des pommes

Petit carpocapse de la pomme

Tétranyque rouge du pommier

A la floraison, apparaissent les premières femelles et les premiers oeufs du Tétranyque rouge du pommier. Ainsi, l'espèce a complété une première génération.

Charançon de za prune

Les adultes de ce ravageur envahissent réellement les pommiers au temps de la pleine floraison. A l'occasion, ils grignotent les pétales des fleurs pour s'alimenter.

Hop Zocampe des pommes

Signalé au Canada en 1940, l'Hoplocampe des pommes, Hoplocampa testudinea (Klug), ne se rencontrait jusqu'à récemment que sur l'île de Vancouver, en Colombie-Britannique. Toutefois, en juin 1979, ce ravageur était trouvé pour la première fois au Québec; au printemps 1980 , on le rencontrait à nouveau dans quelques vergers du sud-ouest de la Province. Cet insecte passe l'hiver à l'état de larves dans le sol et les adultes font leur apparition sur les pommiers au début de la période de floraison. Ceux-ci mesurent environ $6 \mathrm{~mm}$ de longueur; les ailes sont transparentes avec nervures proéminentes foncées. L'abdomen 
et le thorax sont noirs à la partie dorsale et brun jaune à la partie ventrale (P1. III, fig. 11).

La femelle introduit ses oeufs dans le réceptacle des fleurs à la base des sépales. La larve éclot à peu près au temps de la nouaison et pratique une galerie superficielle encerclant une portion du fruit. Par la suite, la larve pénètre au centre du fruit en se creusant un trou de bonne dimension près duquel s'accumulent les excréments. L'espèce est univoltine.

Petit carpocapse de za pomme

Le dépistage du Petit carpocapse de la pomme, Grapholitha pminivora (Walsh), à l'aide d'une phéromone synthétique, indique la présence des premiers adultes dans les vergers à la fin de la période de floraison.

Ces adultes sont des papillons minuscules de couleur terne noirâtre. Sous binoculaire, on note toutefois que les ailes antérieures sont plutôt brun foncé légèrement colorées de brun orangé et de bleu; leur envergure est d'environ $10 \mathrm{~mm}$. Les chenilles à tête brune et au corps blanc rosé mesurent $8 \mathrm{~mm}$ de longueur au terme de leur développement. Elles se nourrissent sur les pommes à la façon de celles du Carpocapse de la pomme, Laspeyresia pomonezza (L.). Les dégâts occasionnés sont très rarement d'importance économique.

\section{CAIICE}

Le stade du calice est atteint lorsque $90 \%$ des pétales des fleurs sont tombées, ce qui a lieu ordinairement durant les derniers fours de mal (P1. I, fig. 8). Les dates extrêmes du calice sont le 20 mal et le 7 juin; la date moyenne, le $29 \mathrm{mal}$. La somme des degrés-jours est alors de 300 unités. 
Date moyenne: 29 mai

Dates extrêmes: 20 mai et 7 juin

Degrés-jours: 300

Ravageurs secondaires:

Carpocapse de la pomme

Cochenille virgule du pommier

Cochenille ostréiforme

Tordeuse à bandes rouges

Campocapse de la ponme (P1. III, figs 6 et 7)

La première capture des adultes du Carpocapse de la pomme, effectuée au moyen d'une phéromone synthétique coInncide le plus souvent avec le stade du calice. A la première capture, la somme des degrés-jours calculée au seuil de $11^{\circ} \mathrm{C}$ et selon la méthode de Baskerville et Emin ${ }^{1}$ est de 110 unités.

Cet insecte est mondialement connu et se classe dans plusieurs pays comme ravageur prépondérant des pommes. Au Québec, durant les deux dernières décennies, il a été toutefois considéré comme ravageur secondaire, étant supplanté par la Mouche de la pomme, Rhagoletis pomonelza (Walsh). Les traitements insecticides appliqués régulièrement contre ce dernier insecte assurent en même temps la répression du Carpocapse et le maintiendrait ainsi au-dessous du seuil de nuisibilité. En fait, dans les vergers négligés, les populations du Carpocapse et les attaques qui en résultent sont très élevées.

1 Baskerville, G.L. et P. Emin. 1969. Rapid estimation of heat accumulation from maximum and minimum temperatures. Ecology 50: 514-517. Trottier, R. 1980. Early warning system for apple pest management in Canada. EPPO BuZz. 10: 253-257. 
Cocheniz? e virgule

La Cochenille virgule, Lepidosaphes ulmi (L.), passe l'hiver à l'état d'oeufs abrités sous de petits boucliers en forme de virgule collés à l'écorce des branches. Ces oeufs éclosent au stade du calice; les larves néonates ne sont mobiles que durant un court laps de temps après quoi elles se fixent à un endroit quelconque sur les brindilles, les branches et quelquefois sur les fruits pour y compléter leur cycle vital et se recouvrir progressivement d'un bouclier. C'est durant le stade mobile de l'insecte qu'il convient d'effectuer un traitement s'il y a lieu.

\section{Cochenizze ostréiforme}

Munie d'un bouclier minuscule de forme ronde légèrement convexe, la Cochenille ostréiforme, Quadraspidotus ostreaeformis (Curt.), se distingue facilement de la Cochenille virgule. L'espèce passerait l'hiver au stade larvaire; mâles et femelles seraient présents dans les vergers au stade du calice et ce serait l'époque désignée pour une intervention. Son cycle évolutif n'a toutefois jamais fait l'objet d'étude au Québec.

Tordeuse à bandes rouges

Les chenilles de cette tordeuse, de couleur vert pâle et à tête jaune paille, sont écloses au stade du calice. Si les populations sont suffisamment abondantes c'est à ce stade qu'il faut appliquer un premier traitement.

\section{NOUAISON}

La nouaison survient environ cinq jours après le stade du calice; à ce moment, les pommes n'ont environ que $6 \mathrm{~mm}$ de diamètre (Pl. I, fig. 9). La date moyenne de la nouaison est le 3 juin, tandis que les dates extrêmes sont le 24 mai et le 14 juin. 


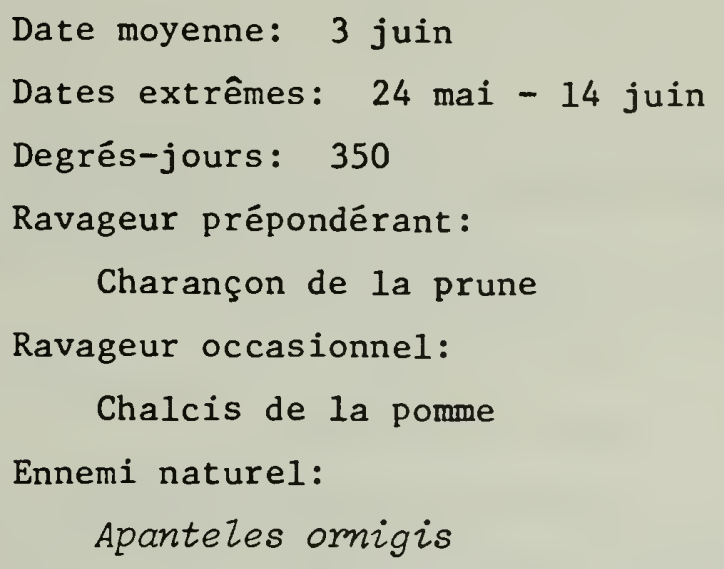

Charançon de la prune

Dès que les fruits sont noués, le Charançon de la prune y dépose ses oeufs marquant le fruit d'une cicatrice en forme de croissant (P1. II, fig. 9). Dans une proportion de $80 \%$, les pontes de ce ravageur ont lieu en juin.

Chalcis de la ponone, Torymus varians (Wlk.)

Ce petit Hyménoptère vert brillant mesurant de 3 à $4 \mathrm{~mm}$ de longueur fait son apparition au temps de la nouaison des fruits et demeure actif durant les trois semaines subséquentes. Les femelles déposent leurs oeufs directement dans les pépins des pommes; la larve se développe à l'intérieur du pépin et y passe l'hiver. Ce chalcis est un ravageur occasionnel et il arrive que ses dégâts soient confondus avec ceux de la Mouche de la pomme.

Apanteles ormigis (Weed) (PI. IV, fig. 10)

Ce braconide joue un rôle de première importance quant à la répression des populations des Mineuses. Au cours des dernières saisons, on a constaté que dans des vergers infestés par la Mineuse marbrée le taux de parasitisme par A. ormigis pouvait atteindre $85 \%$. Les adultes de ce braconide font leur apparition à l'époque de la nouaison. 


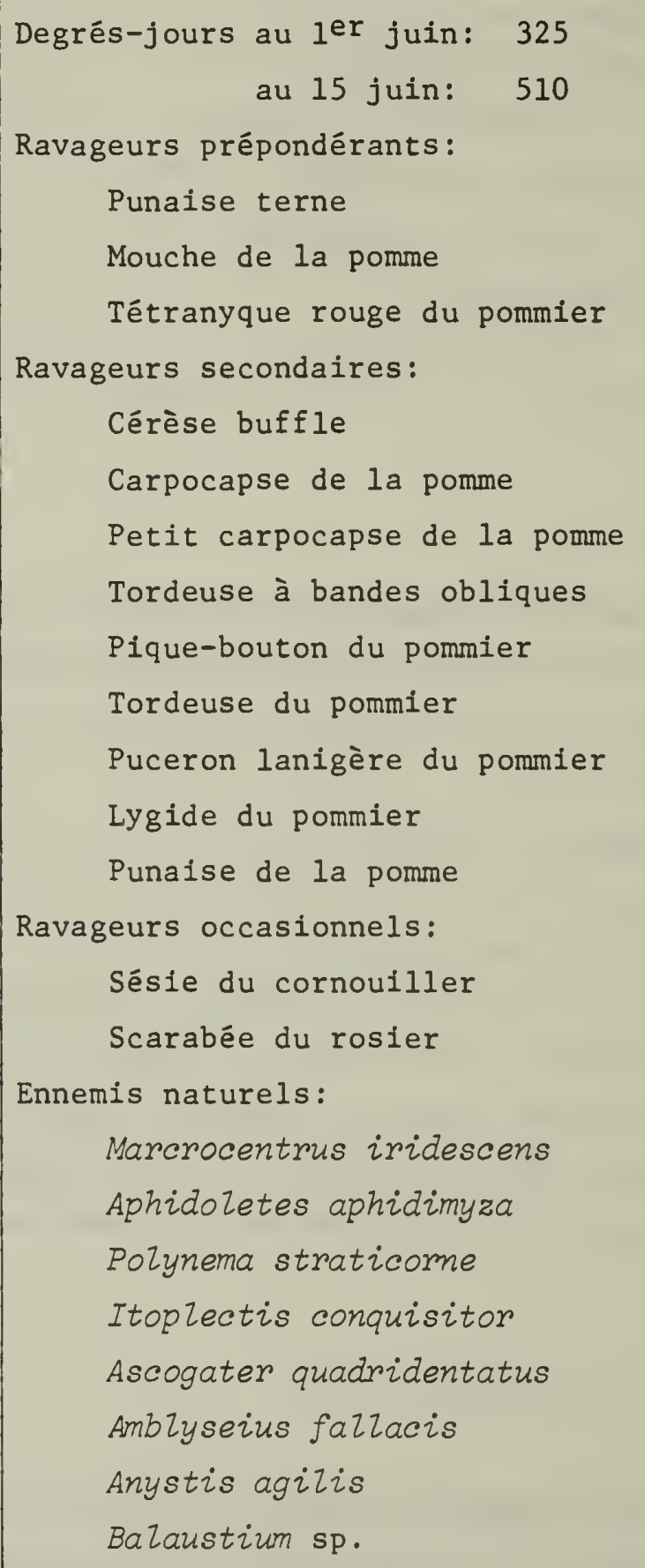


Punaise terme

Au cours de la première quinzaine de juin, la Punaise terne délaisse le pommier pour se confiner aux plantes de couverture.

Mouche de la porme (PI. II, figs 6 et 7)

Un des insectes les plus importants des vergers, la Mouche de la pomme, Rhagoletis pomonelza (Walsh), fait son apparition à la date moyenne du 25 juin (extrêmes: 20 juin et 5 juillet). A ce moment, la somme des degrés-jours est de 450 unités établies à partir d'un seuil de $9^{\circ} \mathrm{C}$ et calculées selon la formule de Baskerville et Emin déjà citée. Depuis plusieurs années, la Mouche de la pomme fait l'objet de dépistage au Québec.

Tétranyque rouge du pommier

La majorité des individus au cours du mois de juin appartiennent aux $2^{\mathrm{e}}$ et $3^{\mathrm{e}}$ générations.

Cérèse buffle

Les oeufs hibernants de la Cérèse buffle éclosent durant la première quinzaine de juin. Les nymphes puis les adultes se nourrissent sur les plantes herbacées, de préférence sur les légumineuses.

Carpocapse de la pome et Petit carpocapse

Les captures de ces deux espèces effectuées à l'aide de phéromones de synthèse demeurent habituellement intensives durant tout le mois de juin. L'éclosion des oeufs du Carpocapse de la pomme survient vers la mi-juin lorsque la somme des degrés-jours est de 210 unités calculées au seuil de $11^{\circ} \mathrm{C}$ et selon $1 \mathrm{a}$ formule de Baskerville et Emin. 
Tordeuse à bandes obliques

Lá courbe des captures des papillons de la Tordeuse à bandes obliques débute entre le 10 et le 15 juin, puis atteint son point maximum à la fin de ce même mois et au début de juillet.

Pique-bouton et Tordeuse du pomier

A l'instar de l'espèce précédente, la présence des adultes de ces deux ravageurs est particulièrement abondante à la fin de juin et au début de juillet.

Puceron Lanigère du pomier

A la mi-juin, on peut déjà noter la présence du Puceron lanigère du pommier, Eriosoma Zanigerum (Hausm.), notamment sur les blessures non complètement cicatrisées résultant de la taille des pommiers.

Lygide du pormier et Punaise de la pomme

Les adultes de ces deux espèces univoltines apparaissent en juin (P1. II, figs 4 et 5 ).

Sésie du comouizzer

Les adultes de la Sésie du cornouiller, Thamosphecia scitula (Harr.), au corps noir marqué de jaune et aux ailes transparentes, sont actifs de la fin de juin à la mi-juillet. La chenille de cette espèce est blanchâtre et se nourrit de sève en demeurant dans des tunnels sous l'écorce de pommiers qui sont déjà affaiblis et sans vigueur. Des vermoulures rougeâtres sur les écorces révèlent sa présence. Depuis 1979, cette espèce fait l'objet d'essais de dépistage. 
Scarabée du rosier

Vers la mi-juin, les adultes du Scarabée du rosier, Macrodactylus subspinosus (F.), envahissent parfois certains vergers plantés à proximité d'endroits très sablonneux. Ces coléoptères, mesurant environ $9 \mathrm{~mm}$ de longueur, de couleur brun jaunâtre et pourvus de longues pattes, grignotent des cavités plus ou moins profondes sur les fruits.

Macrocentrus iridensens French (PI. IV, fig. 6)

En l'absence de traitements insecticides dans un verger expérimental, ce bracomide polyembryonique a, au cours des deux dernières saisons, pratiquement éliminé les larves hibernantes et les larves de la première génération de la Tordeuse à bandes obliques. Cette espèce mérite une attention spéciale.

Aphidoletes aphidimyza (Rondani) (PI. IV, fig. 7)

Cette cécidomyie, qui est un important prédateur du Puceron vert, du Puceron rose et du Puceron lanigère sur le pommier, apparaît au début de juin.

Polynema striaticome Gir.

Il s'agit d'un chalcide dont les adultes apparaissent durant la première quinzaine de juin et qui est un important parasite des oeufs de la Cérèse buffle.

Itoplectis conquisitor (Say) (PI. IV, fig. 8)

Cet endoparasite est très commun s'attaquant à une foule d'insectes, notamment aux tordeuses et au pique-bouton du pommier. 
Ascogaster quadridentatus (Wes.) (PI. IV, fig. 11)

Ce braconide d'environ $4 \mathrm{~mm}$ de longueur attaque les oeufs du Carpocapse de la pomme pour ensuite se développer à l'intérieur de la larve. L'adulte apparaît à la mi-juin au moment des premières éclosions larvaires du Carpocapse. Dans les vergers commerciaux, le taux de parasitisme est d'environ $6 \%$, mais peut atteindre $25 \%$.

Amblyseius fallacis (Gar.) (PI. IV, fig. 12), Anystis agilis Banks et Balaustizon sp.

Les espèces prédatrices s'attaquant au Tétranyque rouge du pommier sont très nombreuses, mais disparaissent dans les vergers commerciaux à cause des traitements insecticides. A. fallacis, A. agilis et Balaustium sp. sont les seules espèces que l'on rencontre en faible nombre dans les vergers commerciaux à partir de la mi-juin.

En 1979, il y a eu un premier essai d'introduction de spécimens d'Amblyseius fallacis résistants à certains insecticides organophosphorés. Les résultats obtenus ont été des plus encourageants.

\section{JUILLET}

Mouche de la pome

L'activité maximale de cet insecte prend place de la mi-juillet à la mi-août.

Tétranyque rouge du pormier

En juillet, la plupart des individus appartiennent aux $3^{\mathrm{e}}$ et $4^{\mathrm{e}}$ générations; les populations atteignent leur densité la plus élevée ordinairement à la fin de juillet et au début d'août. 


Degrés-jours au ler juillet:
au 15 juillet:
Ravageurs prépondérants:
Mouche de la pomme
Tétranyque rouge du pommier
Charançon de la prune
Ravageurs secondaires:
Tordeuse à bandes rouges
Pique-bouton du pommier
Puceron vert du pommier
Ennemis naturels:
Trichogramma minutum
Agathis Zaticinctus
Diadegma obliteratus
Sarcophaga alarichi

Charançon de za prune

Vers la mi-juillet, les larves de ce charançon quittent les pommes et pénètrent dans le sol pour passer successivement aux stades de chrysalides et d'adultes; ces derniers apparaitront surtout durant la $3^{2}$ semaine d'août. La présence des nouveaux adultes se manifeste principalement par une augmentation des piqûres de nutrition (Pl. II, fig. 10).

Tordeuse à bandes rouges

Le début de juillet marque les premières captures des adultes de cette tordeuse qui donneront naissance à la deuxième génération.

Pique-bouton du pormier

Les chenilles de la nouvelle génération qui deviendront les chenilles hibernantes sont abondantes vers 1 e 20 juillet. 
Puceron vert du pomonier

A la fin de juillet, on observe le plus souvent une nette diminution des populations du puceron vert du pommier, ce qui serait dû aux conditions climatiques et à la coriacité du feuillage.

Trichogramma minutum Riley

Dans les vergers de pommiers du Québec, ce parasite cosmopolite a souvent été observé en juiller émergeant des oeufs, entre autres, de la Tordeuse à bandes rouges et du Pique-bouton du pommier.

Agathis laticinctus (Cress.) (PI. IV, fig. 4)

Ce braconide est un parasite très important du Pique-bouton du pommier pouvant réduire de $50 \%$ les populations larvaires. Les adultes apparaissent vers la mi-juillet.

Diadegma obliteratus (Cress.) (PI. IV, fig. 5)

Ce parasite s'attaque aux Tordeuses, aux Olethreutides et aux Mineuses. Sarcophaga aldrichi Park. (PI. IV, fig. 9)

Ce tachinide s'attaque plus particulièrement aux cocons de la Livrée des forêts et peut décimer cette espèce présente dans des vergers négligés.

$$
\text { AOÛT }
$$

Tétranyque rouge du pormier

Cet acarien complète les $4^{\mathrm{e}}$ et $5^{\mathrm{e}}$ générations et, au cours du mols d'août, on peut observer les premiers oeufs d'hibernation déposés sur l'écorce des branches ou dans la cavité oculaire des pommes. 


\section{Degrés-jours au 1er août: 1200 \\ au 15 août: 1450 \\ Ravageur prépondérant: \\ Tétranyque rouge du pommier \\ Ravageurs secondaires: \\ Carpocapse de la pomme \\ Petit carpocapse \\ Cérèse buffle \\ Tordeuse à bandes rouges \\ Puceron lanigère du pommier \\ Eriophyide du pommier}

Carpocapse et Petit carpocapse de la pomme

Au début d'août, apparaissent les papillons de ces deux ravageurs qui donneront naissance à une deuxième génération partielle.

Cérèse buffle

Egalement au début d'août, les femelles de la Cérèse buffle délaissent les plantes herbacées et envahissent les jeunes pommiers. En plus d'implanter leurs oeufs jusque dans le liber des troncs et des brindilles, elles pratiquent deux incisions dans les écorces près de chaque ponte. Lorsqu'elles sont nombreuses, ces blessures peuvent compromettre la croissance des jeunes arbres.

Tordeuse à bandes rouges

L'éclosion des chenilles de la deuxième génération de cette tordeuse est pratiquement complétée au début du mois d'août. 
Puceron Lanigère du pommier

C'est également au début d'août que se manifestent les proliférations du Puceron lanigère qui, depuis quelques années, sont beaucoup plus généralisées.

Eriophyide du pomier

C'est au cours du présent mois qu'apparaissent les premières femelles deutogynes qui passeront l'hiver sous les écailles des bourgeons ou dans les replis d'écorce.

II - PROGRAMME DE LUTTE ET DÉPISTAGE

Dans l'état actuel des choses, on peut difficilement envisager de produire des pommes pour fins commerciales sans recourir à un certain nombre de traitements insecticides. C'est pourquoi nous proposons des traitements de base convenant à l'ensemble des vergers et dirigés contre les ravageurs prépondérants qui sont présentement le Tétranyque rouge, la Mouche de la pomme, les Punaises phytophages, le Charançon de la prune et la Noctuelle du fruit vert. On recommande aussi, s'il y a lieu, des traitements d'appoint ou des traitements spéciaux qui tiennent compte des problèmes locaux et saisonniers.

Les traitements de base comportent une première intervention insecticide effectuée au stade du bouton rose et dirigée contre la Punaise terne et la Noctuelle du fruit vert. Cette intervention peut avoir en même temps une action répressive sur des ravageurs secondaires ou occasionnels présents à cette époque, notamment, les Livrées, la Tordeuse à bandes obliques, la Tordeuse du pommier, les Mineuses, le Pique-bouton et les Pucerons (Tableau I).

Une deuxième intervention, recommandée au stade du calice, est dirigée contre le Charançon de la prune et contre les Punaises; elle peut être 
également efficace contre les Cochenilles, la Tordeuse à bandes rouges, 1 'Hoplocampe des pommes, la Spongieuse et, à nouveau, contre les Pucerons.

Un troisième traitement est effectué contre le Tétranyque rouge du pommier et est appliqué lorsque 1 'on dénombre en moyenne cinq formes mobiles de cet acarien par feuille. Il peut, le cas échéant, être combiné au traitement antérieur ou ultérieur. Cette intervention enraye aussi les populations de l'Eriophyide du pommier et du Tétranyque à deux points.

Un quatrième et un cinquième traitement de base sont effectués respectivement vers le 10 et vers le 25 juillet contre la Mouche de la pomme; ces traitements peuvent être également efficaces contre les Carpocapses, les Pucerons, les Tordeuses et le Pique-bouton du pommier.

TABLEAU 1 Traitements de base dirigés contre les ravageurs prépondérants en regard des ravageurs secondaires ou occasionnels éventuellement présents

\begin{tabular}{|c|c|c|}
\hline $\begin{array}{l}\text { Epoque des } \\
\text { traitements }\end{array}$ & $\begin{array}{l}\text { Ravageurs } \\
\text { prépondérants }\end{array}$ & $\begin{array}{l}\text { Ravageurs secondaires } \\
\text { ou occasionmels }\end{array}$ \\
\hline Bouton rose & $\begin{array}{l}\text { Punaise terne } \\
\text { Noctuelle du fruit } \\
\text { vert }\end{array}$ & $\begin{array}{l}\text { Livrées, Tordeuse à bandes } \\
\text { obliques, Tordeuse du pommier, } \\
\text { Mineuses, Pique-bouton, Pucerons }\end{array}$ \\
\hline Calice & $\begin{array}{l}\text { Charançon de la } \\
\text { prune et Punaises } \\
\text { phytophages }\end{array}$ & $\begin{array}{l}\text { Cochenilles, Tordeuse à bandes } \\
\text { rouges, Pucerons, Hoplocampe, } \\
\text { Spongieuse }\end{array}$ \\
\hline $\begin{array}{l}\text { Juin ou } \\
\text { juillet }\end{array}$ & $\begin{array}{l}\text { Tétranyque rouge } \\
\text { du pommier }\end{array}$ & $\begin{array}{l}\text { Eriophyide du pommier, } \\
\text { Tétranyque à deux points }\end{array}$ \\
\hline 10 juillet & Mouche de la pormme & $\begin{array}{l}\text { Carpocapses, Pucerons, } \\
\text { Tordeuses, Pique-bouton }\end{array}$ \\
\hline 25 juillet & Mouche de la pomme & $\begin{array}{l}\text { Carpocapses, Pucerons, } \\
\text { Tordeuses, Pique-bouton }\end{array}$ \\
\hline
\end{tabular}

Concernant les traitements d'appoint ou spéciaux, leur emploi exige au préalable certaines précautions, car il se peut, par exemple, que les 
populations de Mineuses que $I^{\prime}$ on désire éliminer soient fortement parasitées ou encore que les colonies de pucerons en cause comportent de nombreux prédateurs. Dans ces cas, les interventions insecticides sont non seulement inutiles, mais préjudiciables. C'est alors que la connaissance des principaux parasites et prédateurs, habituellement présents dans les vergers, devient très importante tant pour le conseiller agricole que pour I'arboriculteur lui-même.

Un programme de dépistage s'avère également très utile pour déterminer la présence de tel ou tel ravageur, la densité de la population et la nécessité des traitements. Deux sortes de pièges sont présentement employés avec succès dans certains vergers du Québec:

- le piège à phéromone sexuelle synthétique et

- le piège-appât basé principalement sur l'attractivité de la couleur . Les ravageurs qui font présentement l'objet de piégeage se répartissent comme suit:

Pièges à phéromones

Pièges-appâts

Tordeuse à bandes rouges (1)

Carpocapse de la pomme (1)

Tordeuse à bandes obliques (1)

Mouche de la pomme (1)

Punaise terne (4)

Tordeuse du pommier (1)

Hoplocampe des pommes (4)

Petit carpocapse de la pomme

Sésie du cornouiller (2)

Mineuse marbrée (2)

Noctuelle du fruit vert (3)

Le piège à phéromones renferme une capsule imprégnée d'une substance synthétique comparable à celle émise par la femelle d'une espèce donnée. Cette capsule est placée dans un piège de cargon évasé et pré-englué;

(1) Zolcon Corporation, Palo Alto, California 94304.

(2) Conre1, Needham Heights, Massachusetts 02194.

(3) Prairie Regional Laboratory, NRC Canada, Saskatoon, Sask. S7N OW9.

(4) New England Insect Traps, Colrain, Massachusetts 01340. 
elle peut être efficace sur une superficie d'environ deux hectares durant un mois.

Le piège-appât pour la mouche de la pomme est fait d'un carton jaune $(14 \times 23 \mathrm{~cm})$ pré-englué et pourvu d'un appât à base de sels ammoniacaux. Il doit être placé dans les endroits les plus sujets aux attaques de la Mouche. Le piège-appât pour la Punaise terne et 1'Hoplocampe des pommes est fait d'un carton $(15 \times 20 \mathrm{~cm})$ recouvert de pigments blancs de zinc ne réfléchissant pas les radiations ultraviolettes et enduit d'une glue appelée Tangletrap ${ }^{(R)}$.

Pour la Tordeuse à bandes rouges, le Carpocapse de la pomme, la Tordeuse à bandes obliques et la Mouche de la pomme, mentionnés dans le tableau qui suit, nous sommes en mesure de fournir les dates d'installation des pièges, les périodes de piégeage et le nombre critique de captures à la fin de la période de piégeage:

\begin{tabular}{|c|c|c|c|c|}
\hline$d^{\prime}$ in & $\begin{array}{l}\text { Date } \\
\text { stallation } \\
\text { s pièges }\end{array}$ & & $\begin{array}{l}\text { Périodes } \\
\text { des } \\
\text { captures }\end{array}$ & $\begin{array}{c}\text { Nbre critique } \\
\text { des captures } \\
\text { totales }\end{array}$ \\
\hline $\begin{array}{l}\text { Tordeuse à bandes } \\
\text { rouges }\end{array}$ & 15 avril & 20 & avril - 20 mai & 600 \\
\hline $\begin{array}{l}\text { Carpocapse de } \\
\text { la pomme }\end{array}$ & 15 mai & 20 & mai - 20 juin & 20 \\
\hline $\begin{array}{l}\text { Tordeuse à bandes } \\
\text { obliques }\end{array}$ & $25 \mathrm{mai}$ & 1 & juin - 1 juillet & 100 \\
\hline Tordeuse du pommier & 15 juin & 20 & juin - 20 juillet & 20 \\
\hline $\begin{array}{l}\text { Tordeuse à bandes } \\
\text { rouges ( } 2 \text { e gén.) }\end{array}$ & 20 juin & 25 & juin - 25 juillet & 200 \\
\hline Mouche de la pomme & 20 juin & 25 & juin - 1 septembre & 1 \\
\hline $\begin{array}{l}\text { Tordeuse à handes } \\
\text { obliques ( } 2 \text { e gén. })\end{array}$ & 1 août & 5 & août - 5 septembre & 100 \\
\hline
\end{tabular}

1 Somme des captures de 2 pièges par verger excepté pour la Mouche de la pomme comportant 4 pièges par verger.

Les nombres critiques des captures présentés ici sont provisoires puisqu'ils pourront être ajustés à l'aide de données supplémentaires. 
Les seuils critiques que nous proposons sont provisoires et, pour un bon usage de ces techniques de piégeage, l'arboriculteur fruitier non initié se doit de consulter un conseiller agricole. Pour les espèces qui n'apparaissent pas au tableau, les recherches se continuent afin d'établir avec le plus de précision possible les relations captures-dégâts.

Ce tour d'horizon sur la protection des vergers de pommiers contre ses ravageurs est nécessairement incomplet; il contribue néanmoins à démontrer la complexité des problèmes et les efforts accomplis pour en arriver à une lutte rationnelle plutôt que préventive et empirique.

\section{Remerciements}

Messieurs A. Clément, D. Pitre et L.G. Simard du ministère de l'Agriculture du Canada, ainsi que monsieur J. Touzin du ministère de l'Agriculture, des Pêcheries et de 1'Alimentation du Québec ont, à titre de techniciens, apporté une importante contribution aux travaux de base de cette recherche. De plus, ils ont, avec le concours de monsieur B. Drouin, graphiste au ministère de l'Agriculture, des Pêcheries et de l'Alimentation du Québec, réalisé les planches en couleurs illustrant cet exposé. 

PLANCHE I - Stades phénologiques du développement des pommiers McIntosh

1. Stade dormant

2. Débourrement

3. Débourrement avancé

4. Pré-bouton rose

5. Bouton rose

6. Bouton rose avancé

7. Pleine floraison

8. Calice

9. Nouaison

Photographies:

L.G. Simard 

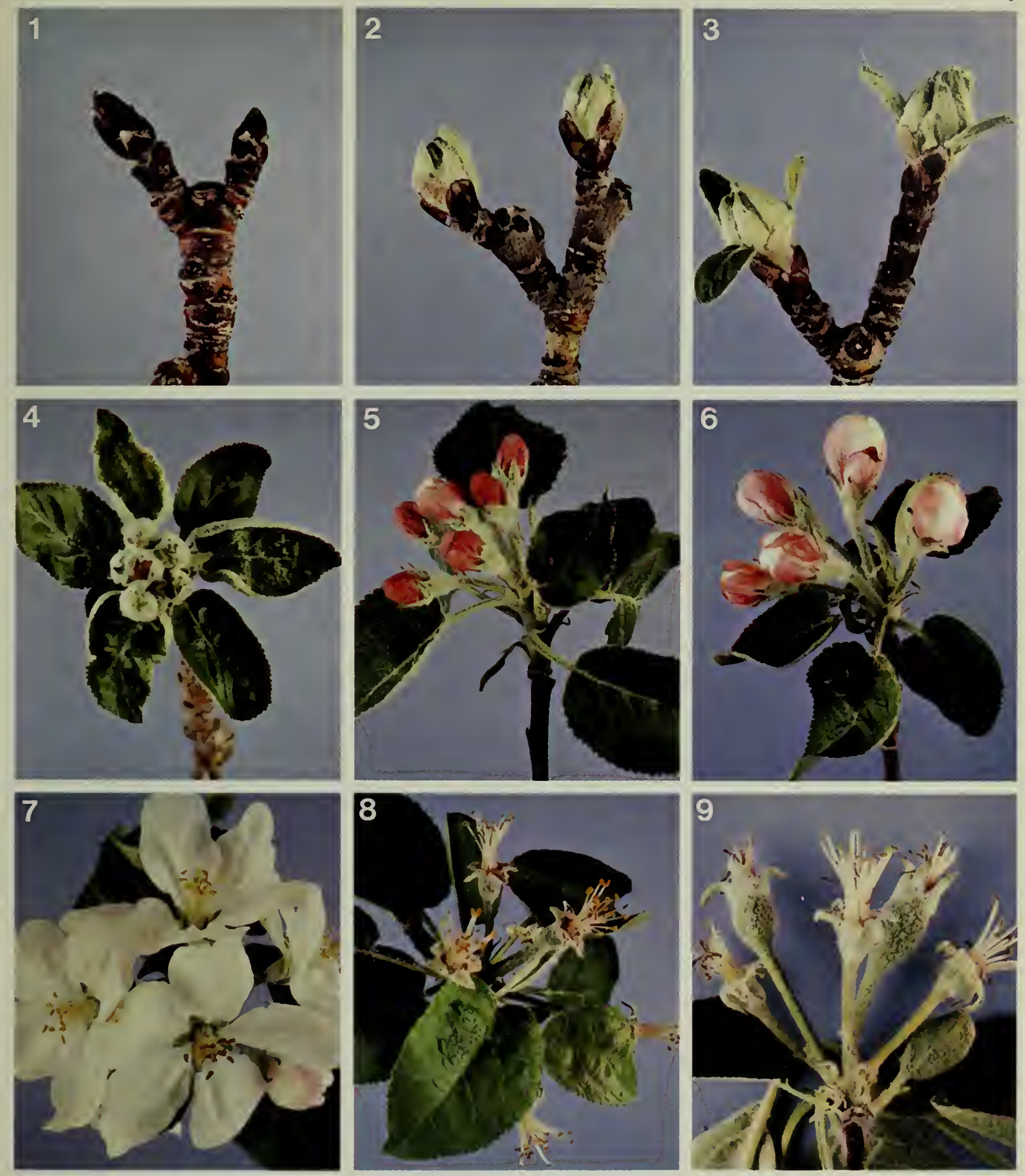


PLANCHE II - Principaux ravageurs des pommiers

1. Femelle du Tétranyque rouge du pommier, Panonychus ulmi (Koch); long. $0,39 \mathrm{~mm}$.

2. Spécimens de l'Eriophyide du pomnier, Aculus schlechtendali (Na1.); long. $0,07 \mathrm{~mm}$.

3. Punaise terne, Lygus lineolaris (P. de B.); long. 5,6 mm.

4. Lygide du pommier, Lygidea mendax Reut; 1ong. 6,6 mm.

5. Punaise de la pomme, Lygocoris communis (Knight); long 5,6 mm.

6. Pommes attaquées par la Mouche de la pomme, Fhagoletis pomonella (Walsh).

7. Mâle de la Mouche de la pomme; long. du corps $5 \mathrm{~mm}$.

8. Adulte du Charançon de la prune, Conotrachelus nenuphar (Hbst.); long. $5 \mathrm{~mm}$.

9. Piqûres de ponte du Charançon de la prune sur pommes fraîchement nouées.

10. Piqûres de nutrition du Charançon de la prune.

11. Chenille de la Noctuelle du fruit vert, Orthosia hibisci (Guenée).

12. Adulte de la Noctuelle du fruit vert; envergure des ailes $40 \mathrm{~mm}$.

13. Dégât cicatrisé sur pomme mûre causé par la chenille de la Noctuelle du fruit vert.

Filotographies:

L.G. Simard, nos $1,2,3,4,6,7,8,10,11,13$

B. Drouin, nos 5,9 

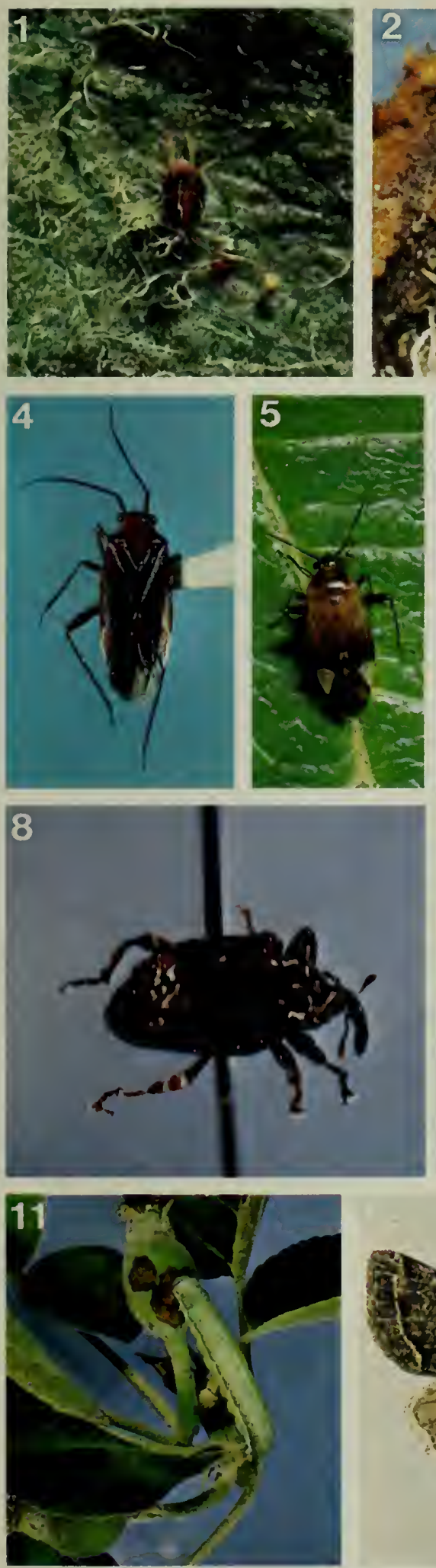
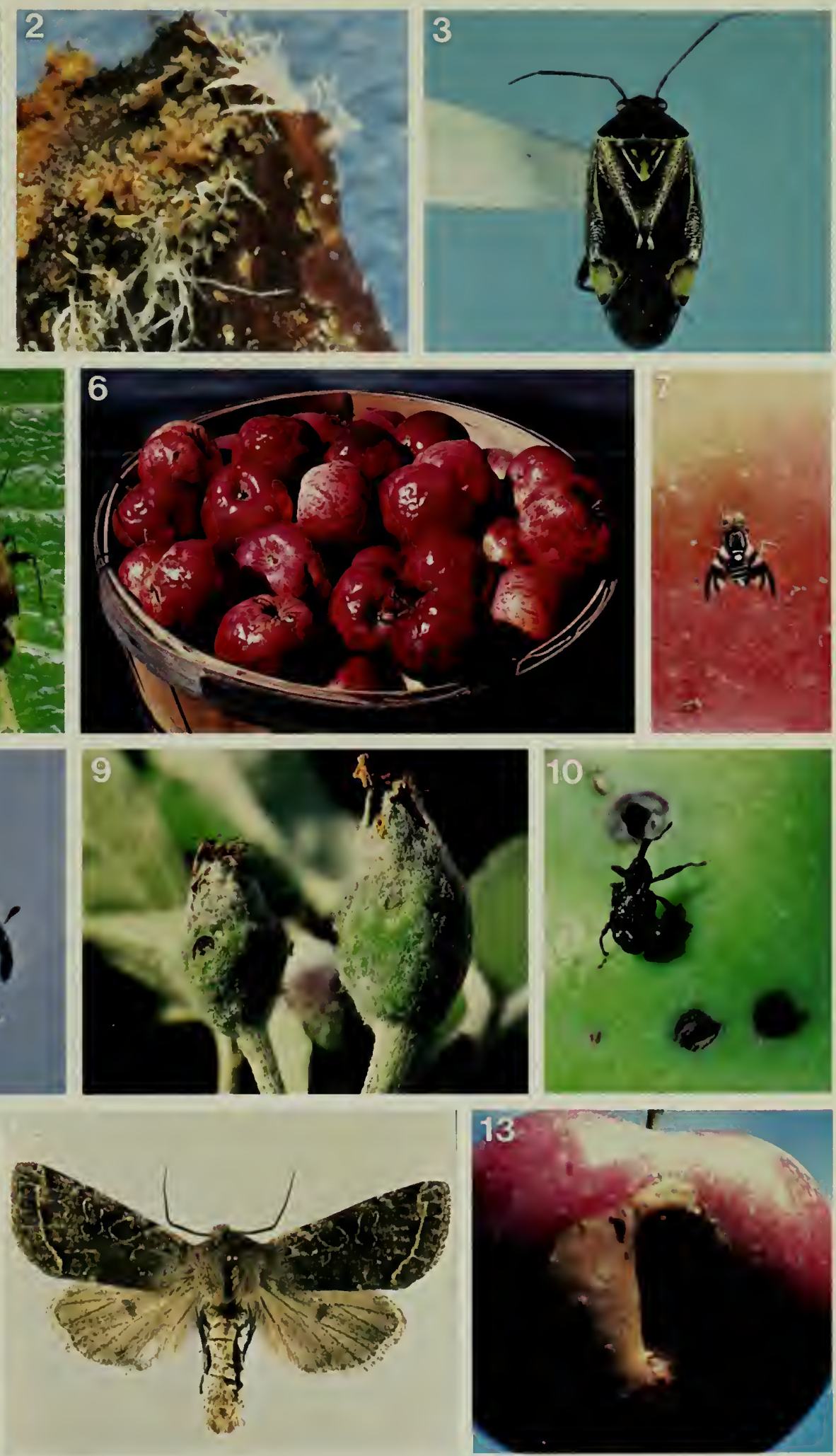

1. Formes aptères du Puceron vert du pommier, Aphis pomi De G.

2. Formes aptères du Puceron rose du pommier, Dysaphis plantaginea (Pass.).

3. Colonies du Puceron rose du pommier sur une pousse terminale.

4. Adulte de la Tordeuse à bandes obliques, Archips rosaceana (Harr.); long. $11,5 \mathrm{~mm}$.

5. Pommes attaquées par la chenille de la Tordeuse à bandes obliques.

6. Adulte du Carpocapse de la pomme, Laspeyresia pomonella (L.); long. $10 \mathrm{~mm}$.

7. Apparence extérieure d'un fruit renfermant la larve du Carpocapse de la pomme.

8. Adulte et chrysalide du Pique-bouton du pomier, Spilonota ocellana (D. \& S.); long. de l'adulte $7,5 \mathrm{~mm}$.

9. Adulte et cuticule nymphale de la Tordeuse du pommier, Archips argyrospilus (WIk.); long. de l'adulte 10,6 mm.

10. Dégâts cicatrisés causés par les chenilles de la Tordeuse du pommier sur des pommes parvenues à maturité.

11. Adulte de l'Hoplocampe des pommes Hoplocampa testudinea (Klug); long. $6 \mathrm{~mm}$.

Photographies:

B. Drouin, nos $1,2,3,8,11$

L.C. Simard, nos $4,5,6,7,9,10$ 

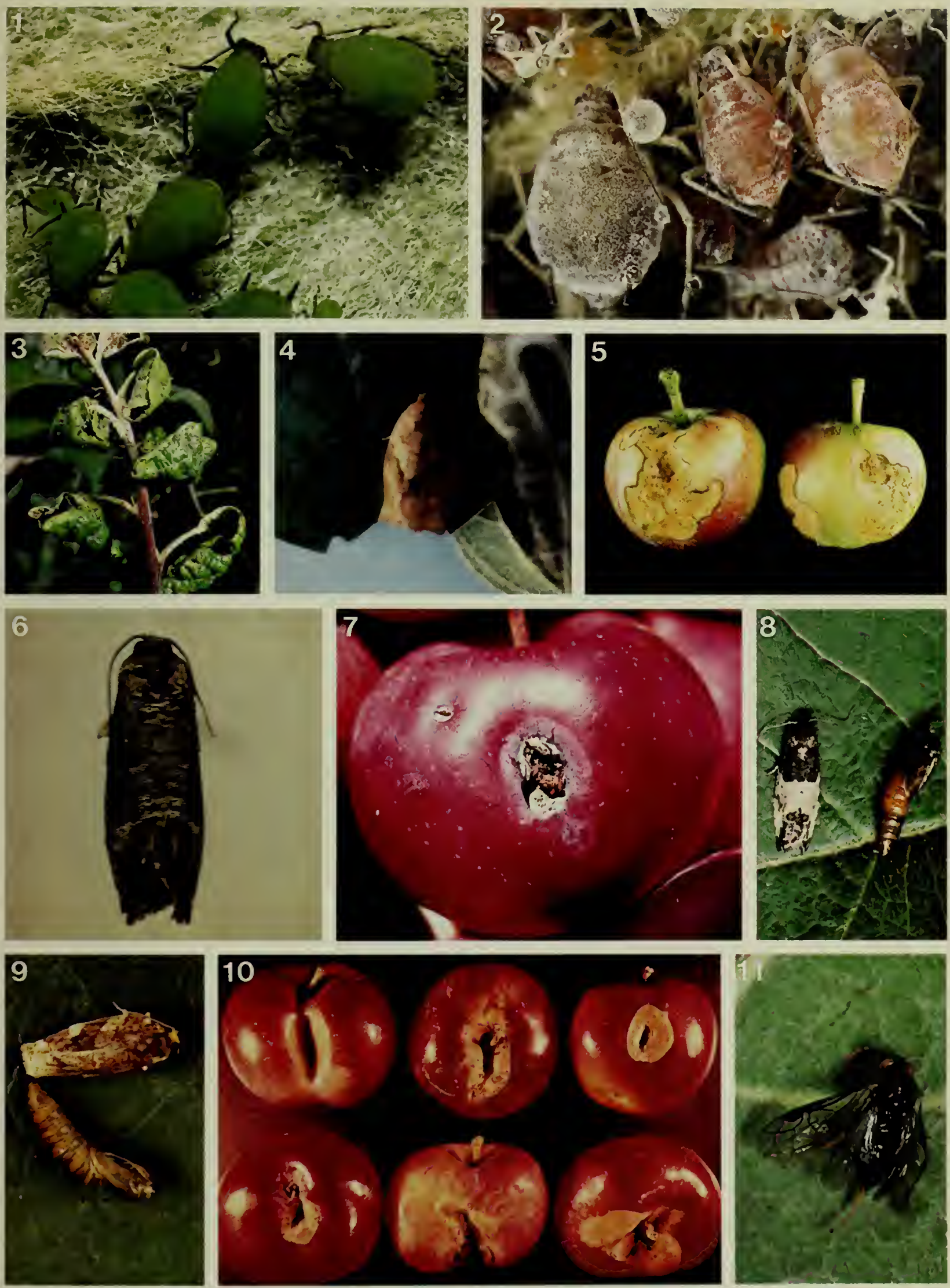

PLANCHE IV - Certains ennemis naturels des ravageurs des pommiers

1. AlZothrombizon Zerouxii Moss; 1ong. 3,3 mm.

2. Oeuf de Syrphide; long. $1 \mathrm{~mm}$.

3. Larve de Syrphide.

3a. Coccinelles prédatrices.

4. Agathis laticinctus (Cresson); long. 4,2 mm.

5. Diadegma obliteratus (Cresson); long. 4,5 mm.

6. Macrocentms iridescens French; 1ong. 3,2 mm.

7. Aphidoletes aphidimyza (Rondan1); 1ong. 1,8 mm.

8. Itoplectis conquisitor (Say); long. $8,2 \mathrm{~mm}$.

9. Sarcophaga aldrichi (Park.); Iong. 4,3 mm.

10. Apanteles ornigis (Weed); 1ong. 2,3 mm.

11. Ascogaster quadridentatus Wes.; Iong. 4,3 $\mathrm{mm}$.

12. Colonie d'Amblyseius fazzacis (Garman); 1ong. de 1'adulte, 0,32 mm.

Priotographies:

B. Drouin, no 1

J. Touzin, nos $2,3,3 a$

L.G. Simard, nos $4,5,6,7,8,9,10,11,12$ 

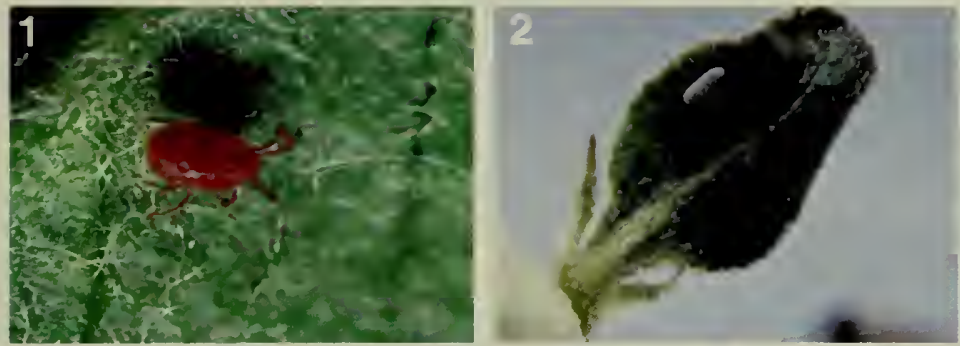
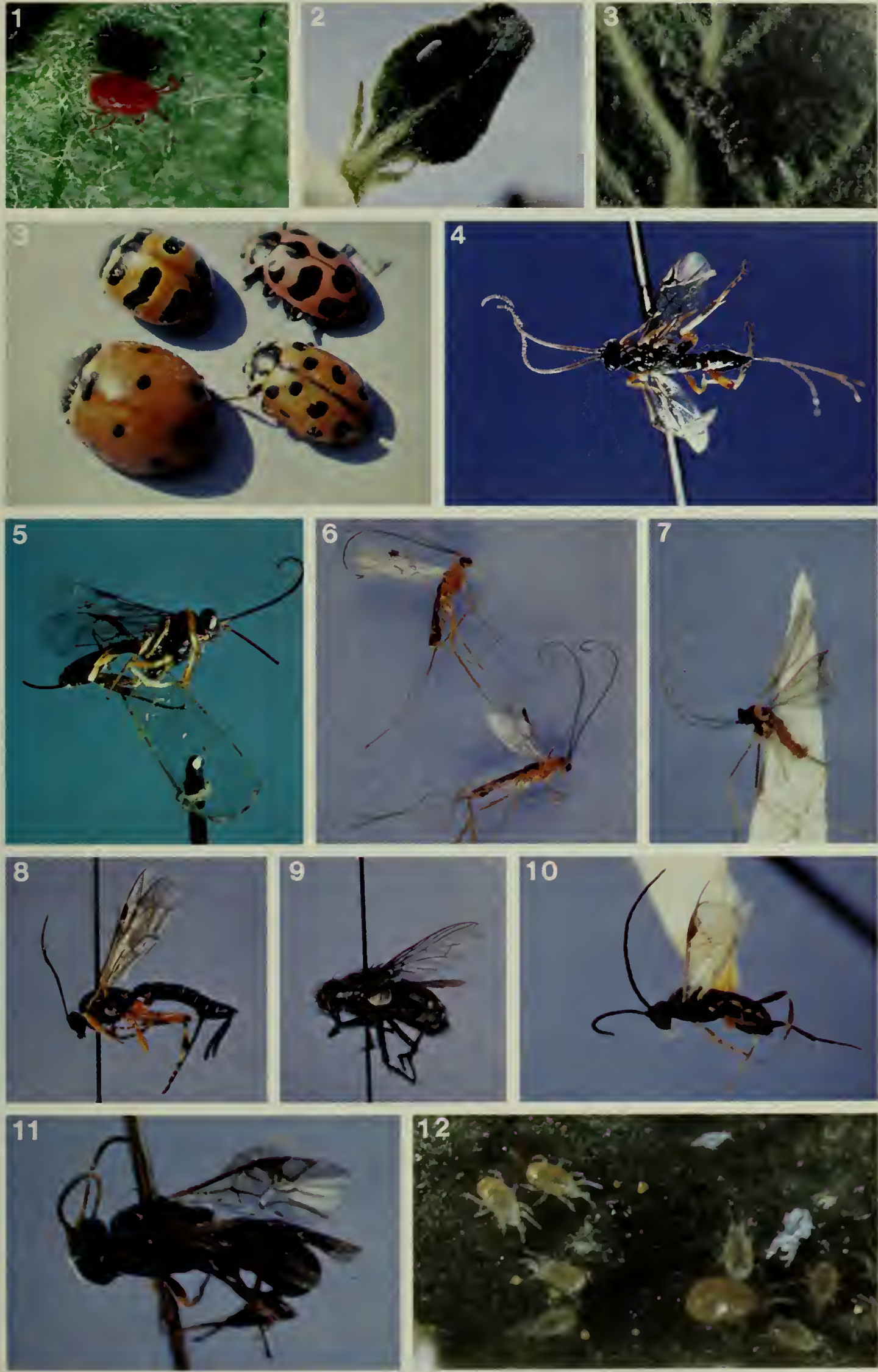

INDEX

Page Page

Aculus schlechtendali voir

coccinelles.......5, 8; P1.IV, fig.3a

Eriophyide du pommier. Cochenille ostréiforme........17, 18 Agathis Zaticinctus.............. Cochenille virgule. $.17,18$

...........25, 26; P1.IV, fig.4 ConotracheZus nenuphar voir Charançon Azzothrombium Zerouxii.............. de la prune.

............... 4; P1.IV, fig.1 Ctenicera spp. voir Taupins......... Amblyseius fazlacis............... débourrement..........4; Pl.I, fig.2 20, 24; P1.IV, fig.12 débourrement avancé.....8; P1.I, fig.3 Anystis agizis............. 2n, 24 degrés-jours...2,.3, 5, 8, 9, . 10, 11 Apanteles omigis...19; P1.IV, fig.10 Aphidoletes aphidimyza.

$13,14,15,16,17,19,20,25,27$

...........20, 23; P1.IV, fig.7 Diadegma obziteratus....................................

Aphis pomi voir Puceron vert du

pommier.

Archips argyrospizus voir Tordeuse

du pommier.

Archips rosaceana voir Tordeuse à

bandes obliques. Argyrotaenia veZutinana voir Tordeuse

à bandes rouges................ Ascogaster quadridentatus.

.20, 24; P1.IV, fig.11

Balaustium sp............20, 24 bouton rose.........11; P1.I, fig.5 bouton rose avancé....13; P1.I, fig.6 Hoplocampe des pommes.............. calice..............16; P1.I, fig.8 Carpocapse de la pomme.16, 17, 2n, 21 $27,29,30,31 ; \mathrm{P} 1$.III, figs 6 et 7 Cérèse buffle...........20,21, 27 Chalcis du pommier. .19 Charançon de la prune.

$\ldots \ldots \ldots \ldots \ldots \ldots \ldots \ldots 1,11,12,15$ 25, 28, 29; P1.II, figs 8, 9 et 10 Lithocolzetis blancardeZza voir Mineuse
Dysaphis plantaginea voir Puceron rose du pommier.

ennemis naturels...2, 3, 5, 19, 20, 25 Eriophyide du pommier.............. $\ldots \ldots 5,6,27,28,29 ;$ Pl.II, fig.2 Eriosoma Zanigemum voir Puceron

lanigère du pommier.

floraison............14, P1.I, fig.7 Grapholitha prunivora voir Petit

carpocapse de la pomme........... Hoplocampa testudinea voir Hoplocampe des pommes.................... ........15, 30, 31; P1.III, fig.11

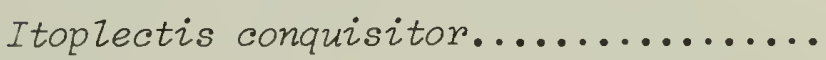
...........20, 23; P1.IV, fig.8 Laspeyresia pomonezla voir Carpocapse de la pomme

Lepidosaphes uZmi voir Cochenille

virgule.................... marbrée. 
Lithocolletis crataegella.........9 Lithocolletis malimalifoliella.....99 Lithocolletis scuderella..........99 Livrée d'Amérique.............5, 7 Livrée des forêts............. 7 Livrées...................28, 29 Lygide du pommier................ .....13, 14, 20, 22; P1.II, fig.4 Lygidea mendax voir Lygide du

pommier. Lygocoris communis voir Punaise

de la pomme.

Lygus lineolaris voir Punaise terne.. Macrocentmus iridescens............. $.20,23 ;$ P1.IV, fig. 6 Macrodactylus subspinosus voir

Scarabée du rosier............... MaZacosoma americanum voir Livrée

d'Amérique.................... Malacosoma disstria voir Livrée

des forêts................... Mineuse marbrée...........8, 9, 30 Mineuses des feuilles.........28, 29 Mouche de la pomme................ $\ldots \ldots \ldots \ldots 1,17,20,21,24,25$ $28,29,30,31$; PI.II, figs 6 et 7 Noctuelle du fruit vert. $.1,3,10,28$ 29, 30; P1.II, figs 11,12 et 13 nouaison...........18; P1.I, fig.9 Orthosia hibisci voir Noctuelle du

fruit vert.................... Panonychus ulmi voir Tétranyque rouge Rhagoletis pomonelza voir Mouche de

du pommier.....................

Petit carpocapse de la pomme........ $\ldots \ldots \ldots 15,16,20,21,27,29,30$ pièges à phéromones............30, 31 pièges-appâts................30, 31 Pique-bouton du pommier.........5, 6 20, 22, 25, 28, 29; P1.III, fig. 8 Polynema straticorme..........20, 23 Lymantria dispar voir Spongieuse..... Pré-bouton rose........10; P1.I, fig.4 Pseudexentera mali voir Tordeuse pâle du pommier.

Pucerons des graminées.......... 7 Puceron lanigère du pommier. $20,22,27,28$

Puceron rose du pommier............. 8,$9 ;$ P1. III,figs 2 et 3 Puceron vert du pommier. .....10, 11, 25, 26; P1.III, fig.1

Pucerons...................28, 29 Punaise de la pomme.

.....13, 14, 20, 22; P1.II, fig.5 Punaise terne................. 4 5, 20, 21, 28, 30, 31; Pl.II, fig.3 Punaises phytophages.........1, 28, 29 Quadraspidotus ostreaeformis voir

Cochenille ostréiforme............ ravageurs occasionnels.............. $\ldots \ldots 2,3,5,9,13,15,19,20,29$ ravageurs prépondérants.............. $\ldots \ldots \ldots 1,2,3,5,10,11,15,19$ $20,25,27,28,29$;P1.II, figs 1-13

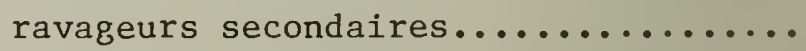
$.2,5,9,10,11,13,17$ 20, 25, 27, 29; P1.III, figs 1- 11 la pomme...................... Rhopalosiphum fitchii voir Puceron des graminées. 
Page

Page

Sarcophaga azdrichi.............. $.25,26 ; \mathrm{Pl} . \mathrm{IV}$, fig.9

Tetranychus urticae volr Tétranyque

à deux points.

Scarabée du rosier..........2.0, 23

Sésie du cornouiller......20, 22, 30

Spizonata ocelzana voir Pique-bouton

du pommier.

Spongieuse..............8, 9, 10

stade dormant.......3, 4; Pl.I, fig.1 syrphes......5, 7; P1.4, figs 2 et 3 Taeniothrips inconsequens voir Thrips

du poirier....................

taupins................... 7

Tavelure du pommier............4

Tétranyque à deux points.......8, 9

Tétranyque rouge du pomier.........

$\ldots \ldots \ldots \ldots 1,11,12,15,20,21,24$

$25,26,27,28,29 ;$ Pl.II, fig. 1

Thamosphecia scitula voir Sésie du

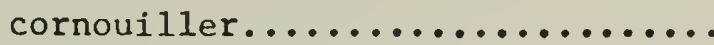

Thrips du poirier............13, 14

Tordeuse à bandes obliques..........

...........5,6, 7, 18, 20, 22

$28,29,30,31 ; \mathrm{P} 1$. III, figs 4 et 5

Tordeuse à bandes rouges............

$\ldots \ldots \ldots \ldots, 6,25,27,29,30,31$

Tordeuse du pommier.......11, 12, 20

$22,28,29$; P1. III, figs 9 et 10

Tordeuse pâle du pommier........3, 4

Torymus varians voir Chalcis du

pommier....................

Trichograrma minutum.........25, 26

Venturia inaequalis voir tavelure du

pommier 



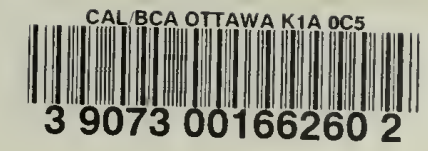


